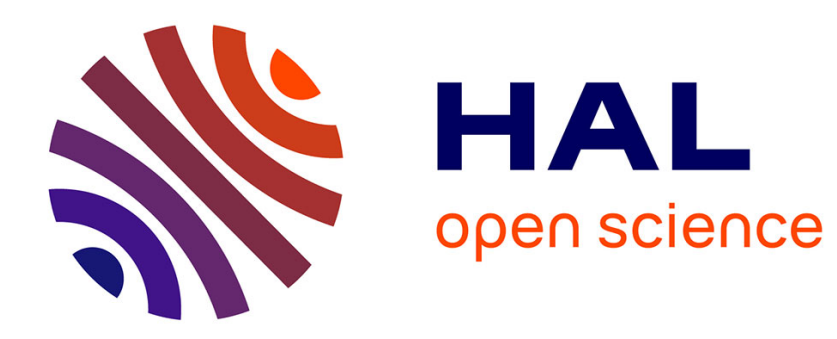

\title{
Error analysis of the compliance model for the Signorini problem
}

Pierre Cantin, Patrick Hild

\section{To cite this version:}

Pierre Cantin, Patrick Hild. Error analysis of the compliance model for the Signorini problem. 2020. hal-02494031

\section{HAL Id: hal-02494031 \\ https://hal.science/hal-02494031}

Preprint submitted on 28 Feb 2020

HAL is a multi-disciplinary open access archive for the deposit and dissemination of scientific research documents, whether they are published or not. The documents may come from teaching and research institutions in France or abroad, or from public or private research centers.
L'archive ouverte pluridisciplinaire HAL, est destinée au dépôt et à la diffusion de documents scientifiques de niveau recherche, publiés ou non, émanant des établissements d'enseignement et de recherche français ou étrangers, des laboratoires publics ou privés. 


\title{
Error analysis of the compliance model for the Signorini problem
}

\author{
Pierre Cantin ${ }^{1}$ and Patrick Hild ${ }^{1}$ \\ ${ }^{1}$ Institut de Mathématiques de Toulouse - UMR CNRS 5219, Université Paul Sabatier, France
}

\begin{abstract}
The present paper is concerned with a class of penalized Signorini problems also called normal compliance models. These nonlinear models approximate the Signorini problem and are characterized both by a penalty parameter $\varepsilon$ and by a "power parameter" $\alpha \geq 1$, where $\alpha=1$ corresponds to the standard penalization. We choose a continuous conforming linear finite element approximation in space dimensions $d=2,3$ to obtain an $L^{2}$-error estimate of order $h^{2}$ when $d=2, \alpha=2, \varepsilon \geq \theta h$ ( $\theta$ large enough) and when the solution is $W^{2,3}$-regular. A similar estimate is obtained when $d=3$ under slightly more restrictive assumptions on $\varepsilon$.
\end{abstract}

\section{Introduction}

Penalty methods are classical and widespread tools for the numerical treatment of constrained problems, such as the unilateral contact (Signorini problem) arising in mechanics of deformable bodies, where a nonlinear boundary condition is written as an inequality. The main idea of penalization is to replace the inequality constraint by a penalized non linear equality that becomes larger as the solution fails to satisfy this inequality constraint.

For the Signorini problem, the penalty method is well-known and is generally used when approximating this problem using finite elements. The convergence analysis of the penalized models was first considered in the 80 's, see $[16,22]$ and the references therein. In the last ten years, some new results dealing with $H^{1}$-error estimates have been obtained for standard penalization in $[7]$ and more recently in $[12,11]$. To summarize the current state of the art, the convergence of the discrete penalized solution towards both the solution to the penalized problem and of the Signorini problem are optimal in two and three dimensions when using standard linear finite elements provided that the penalty parameter $\varepsilon$ behaves like $h$ (up to a constant), representing the mesh-size. These recent results complete the existing $H^{1}$-optimal estimates for Signorini problem using other discrete models such as the variational inequality [13], Lagrange multiplier methods [5, 14] or Nitsche methods [4, 6, 8].

Concerning the $L^{2}$-error estimates for Signorini-like problems, the analysis becomes really more difficult. The main reason is the standard Aubin-Nitsche technique well suited for linear problems can not be applied in its original form, neither for the Signorini problem nor for 
the penalized formulation, due to a lack of Galerkin orthogonality. Most of the efforts to obtain $L^{2}$-error estimate have been devoted to the Signorini problem written as a variational inequality, but as far as we know there are very few results. We mention the early works in [27] used in [10] where a complicated adjoint problem is involved and whose regularity is not established. Using linear finite elements, we also refer to the recent works [9] where an $L^{4}$-estimate of order $2-\varepsilon$ is obtained in two dimensions with additional assumptions on the contact sets, and [30] where authors prove a $3 / 2-\varepsilon$ error estimate in the $L^{2}$-norm when the solution is $H^{5 / 2-\varepsilon}$-regular. Besides, the numerous numerical results dealing with $L^{2}$-error estimates (see, e.g., $[9,20,21,30]$ ) indicate that the convergences behave well and are optimal, so the main lack comes from the mathematical analysis.

In this work, we are interested in a generalized class of penalty models applied to the Signorini problem, the so-called normal compliance model, corresponding to a power-law regularization of the penalty methods. This model was introduced and studied in [25, 28, 23, 24 ], in the context of friction phenomena between a rigid body with a rough and deformable body. Applied to the Signorini problem, the boundary contact condition on a portion $\Gamma_{C}$ of the boundary $\partial \Omega$ is $\varepsilon \partial_{n} u=-[u]_{+}^{\alpha}$, where $[u]_{+}$represents the positive part of the solution $u: \Omega \rightarrow \mathbb{R}, \varepsilon>0$ is the penalty parameter, and $\alpha \geq 1$ is the regularization parameter. The compliance model for the Signorini problem that we consider in this work is then: find $u: \Omega \rightarrow \mathbb{R}$ such that

$$
-\Delta u=f \quad \text { in } \Omega, \quad u=0 \quad \text { on } \Gamma_{D}, \quad \varepsilon \partial_{n} u+[u]_{+}^{\alpha}=0 \quad \text { on } \Gamma_{C}=\partial \Omega \backslash \Gamma_{D} .
$$

When $\alpha=1$, the boundary condition on the contact boundary $\Gamma_{C}$ becomes $\varepsilon \partial_{n} u=-[u]_{+}$, corresponding to the classical penalized Signorini problem.

The main motivation of considering the normal compliance model (1) with $\alpha>1$ is that the map $q_{\alpha}: u \mapsto[u]_{+}^{\alpha}$ is now differentiable. In our analysis, this property is essential to obtain optimal $L^{2}$-error estimates. As in the linear case using the Aubin-Nitsche lemma, our $L^{2}$-error analysis is based on the introduction of a companion problem, but different from the adjoint problem. Taking inspiration from the extension of the Aubin-Nitsche trick for semilinear problems developed in [19], the companion problem is defined by differentiating $q_{\alpha}$ in (1), so that we replace the Galerkin orthogonality argument used in the linear case by a first order Taylor expansion of $q_{\alpha}$. Combining this approach with a fine $\varepsilon$-robust a priori error analysis in energy norm, we then obtain optimal error estimate in $L^{2}$-norm when the parameters $\varepsilon$ and $h$ satisfy some explicit conditions.

Our work contains two major contributions concerning the error analysis using linear finite elements, in two and three space dimension, for the compliance model of the Signorini problem (1). The first one concerns the a priori error analysis in energy norm; for all suitable power-law parameters $\alpha \geq 1$, we obtain optimal error estimates, robust with respect to $\varepsilon$ under the condition that $h / \varepsilon$ is bounded. The second contribution is the optimal $L^{2}$-error estimate when $\alpha=2$. Assuming that the exact solution is $W^{2,3}$-regular, we prove that $\left\|u-u_{h}\right\|_{L^{2}} \leq c h^{2}$ if $\varepsilon \geq \theta h$ (with $\theta$ large enough) in two space dimension or $\varepsilon \geq \theta \sqrt{h}$ in three space dimension. We also extend these results when $\alpha>2$.

The paper is organized as follows. Section 2 deals with the formulation of the compliance model for the Signorini problem, its associated weak form, the existence and uniqueness results and some a priori estimates. Section 3 uses the most common approximation with 
continuous linear finite elements, and $H^{1}$-error estimates of order $h$ are obtained when $h / \varepsilon$ is bounded and the solution is $W^{2, \alpha+1}$-regular. In section 4 we prove $L^{2}$-error estimates of order 2 in two and three space dimensions when $\alpha \geq 2$. Section 5 contains some technical lemmata used in the analysis.

\section{The compliance model for the Signorini problem}

Considering $f \in L^{2}(\Omega)$ and $\varepsilon>0$, we are studying the existence and the uniqueness of $u_{\varepsilon}: \Omega \subset \mathbb{R}^{d} \rightarrow \mathbb{R}$ with $d \in\{2,3\}$, such that

$$
\begin{aligned}
& -\Delta u_{\varepsilon}=f \text { in } \Omega, \\
& u_{\varepsilon}=0 \text { on } \Gamma_{D}, \\
& \varepsilon \partial_{n} u_{\varepsilon}+\left[u_{\varepsilon}\right]_{+}^{\alpha}=0 \text { on } \Gamma_{C} \text {, }
\end{aligned}
$$

where the boundary of $\Omega$, denoted by $\partial \Omega$ is exactly divided into $\Gamma_{C}$ and $\Gamma_{D}$, i.e., $\partial \Omega=\Gamma_{D} \cup \Gamma_{C}$ and $\Gamma_{D} \cap \Gamma_{C}=\emptyset$. The parameter $\alpha \geq 1$ is fixed and we denote by $[t]_{+}=\frac{1}{2}(t+|t|)$ the positive part of any $t \in \mathbb{R}$.

We set $V=\left\{v: v \in H^{1}(\Omega), v=0\right.$ on $\left.\Gamma_{D}\right\}$. The well-posedness analysis of $(2)$ is performed by considering the equivalent variational formulation:

$$
u_{\varepsilon} \in V \quad: \quad a\left(u_{\varepsilon}, v\right)+\frac{1}{\varepsilon} b\left(u_{\varepsilon}, v\right)=\int_{\Omega} f v, \quad \forall v \in V
$$

where we denote, for all $v, w \in V$,

$$
a(v, w)=\int_{\Omega} \nabla v \cdot \nabla w \quad \text { and } \quad b(v, w)=\int_{\Gamma_{C}} q_{\alpha}(v) w
$$

with $q_{\alpha}: t \in \mathbb{R} \rightarrow[t]_{+}^{\alpha}$. To lighten the notation, we do not write the $d \Omega$ and $d \Gamma$ terms in the integrals. In the rest of the paper, we will assume that

$$
\alpha \in[1, \infty) \text { if } d=2 \text { and } \alpha \in[1,3] \text { if } d=3 \text {, }
$$

so that the term $b(v, w)$ is well-defined for all $v, w \in V$ owing to the continuity of the trace map $H^{1}(\Omega) \rightarrow L^{\alpha+1}(\partial \Omega)$.

\subsection{Well-posedness}

The main ingredient to prove the well-posedness of (3) is to prove that $b$ is strongly monotone on $V$, which is a consequence of the following proposition.

Proposition 2.1. For all $v, w \in L^{\alpha+1}\left(\Gamma_{C}\right)$,

$$
b(v, v-w)-b(w, v-w) \geq c\left\|[v]_{+}-[w]_{+}\right\|_{L^{\alpha+1}\left(\Gamma_{C}\right)}^{\alpha+1},
$$

with $c>0$ independent of $v$ and $w$. 
Proof. For all $s, t \in \mathbb{R}$, we have from [17] the estimate $\left(t|t|^{\alpha-1}-s|s|^{\alpha-1}\right)(t-s) \geq c|t-s|^{\alpha+1}$ with $c>0$ independent of $t, s$. Taking $t=[v]_{+}$and $s=[w]_{+}$yields the result.

It then follows the strong monotonicity of

$$
T: V \rightarrow V^{\prime} \quad: \quad\langle T(v), w\rangle_{V^{\prime}, V}=a(v, w)+\frac{1}{\varepsilon} b(v, w) \quad \forall v, w \in V .
$$

Lemma 2.2. For all $v, w \in V$,

$$
\langle T(v), v-w\rangle_{V^{\prime}, V}-\langle T(w), v-w\rangle_{V^{\prime}, V} \gtrsim|v-w|_{H^{1}(\Omega)}^{2}+\frac{1}{\varepsilon}\left\|[v]_{+}-[w]_{+}\right\|_{L^{\alpha+1}\left(\Gamma_{C}\right)}^{\alpha+1} .
$$

To alleviate the notations, we denote by $A \lesssim B$ the estimate $A \leq c B$ when $c>0$ is some non relevant constant, independent of the problem parameters. The notation $A \sim B$ means that $A \lesssim B$ and $B \lesssim A$ hold simultaneously.

Lemma 2.3. For all $v, w, z \in V$,

$$
\begin{aligned}
\langle T(v), z\rangle_{V^{\prime}, V}-\langle T(w), z\rangle_{V^{\prime}, V} & \lesssim|v-w|_{H^{1}(\Omega)}|z|_{H^{1}(\Omega)} \\
+ & \frac{1}{\varepsilon}\|v-w\|_{L^{\alpha+1}\left(\Gamma_{C}\right)}\|z\|_{L^{\alpha+1}\left(\Gamma_{C}\right)}\left(\left\|[v]_{+}\right\|_{L^{\alpha+1}\left(\Gamma_{C}\right)}^{\alpha-1}+\left\|[w]_{+}\right\|_{L^{\alpha+1}\left(\Gamma_{C}\right)}^{\alpha-1}\right) .
\end{aligned}
$$

Hence, $T: V \rightarrow V^{\prime}$ is continuous.

Proof. From the definition of $T$, it suffices to prove that for all $v, w, z \in V$,

$$
|b(v, z)-b(w, z)| \lesssim\|v-w\|_{L^{\alpha+1}\left(\Gamma_{C}\right)}\|z\|_{L^{\alpha+1}\left(\Gamma_{C}\right)}\left(\left\|[v]_{+}\right\|_{L^{\alpha+1}\left(\Gamma_{C}\right)}^{\alpha-1}+\left\|[w]_{+}\right\|_{L^{\alpha+1}\left(\Gamma_{C}\right)}^{\alpha-1}\right) .
$$

Observing that $\left|[x]_{+}^{\alpha}-[y]_{+}^{\alpha}\right| \leq c\left|[x]_{+}-[y]_{+}\right|\left([x]_{+}+[y]_{+}\right)^{\alpha-1}$ for all $x, y \in \mathbb{R}$, with $c>0$ from [17], it follows by Hölder inequality

$$
\int_{\Gamma_{C}}\left|\left([v]_{+}^{\alpha}-[w]_{+}^{\alpha}\right) z\right| \lesssim\left\|[v]_{+}-[w]_{+}\right\|_{L^{\alpha+1}\left(\Gamma_{C}\right)}\|z\|_{L^{\alpha+1}\left(\Gamma_{C}\right)}\left(\int_{\Gamma_{C}}\left([v]_{+}+[w]_{+}\right)^{(\alpha-1) p}\right)^{\frac{1}{p}}
$$

with $\frac{1}{p}+\frac{2}{\alpha+1}=1$, i.e. $p(\alpha-1)=\alpha+1$. Then, the estimate (6) follows using the triangular inequality.

Strong monotonicity and continuity of $T$ obtained in Lemmata 2.2 and 2.3, respectively, then gives the well-posedness of (3).

Theorem 2.4. For all $f \in L^{2}(\Omega)$, problem (3) admits an unique solution $u_{\varepsilon} \in V$ and there exists $c>0$, independent of $\varepsilon$ and $u_{\varepsilon}$, such that

$$
\left|u_{\varepsilon}\right|_{H^{1}(\Omega)}^{2}+\frac{1}{\varepsilon}\left\|\left[u_{\varepsilon}\right]_{+}\right\|_{L^{\alpha+1}\left(\Gamma_{C}\right)}^{\alpha+1} \leq c\|f\|_{L^{2}(\Omega)}^{2} .
$$




\subsection{Convergence analysis}

In this section, we recall the convergence result of $u_{\varepsilon}$ to the Signorini solution $u_{S}$ when $\varepsilon \rightarrow 0$. This result, obtained in [16] for $\alpha=1$, remains valid if $\alpha$ satisfies (5).

Lemma 2.5. Let $u_{\varepsilon} \in V$ be the exact solution of (3). Then, $u_{\varepsilon} \rightarrow u_{S}$ in $V$ where $u_{S} \in$ $K:=\left\{v \in V \mid v \leq 0\right.$ on $\left.\Gamma_{C}\right\}$ is the unique solution solving

$$
a\left(u_{S}, w-u_{S}\right) \geq\left(f, w-u_{S}\right), \quad \forall w \in K .
$$

Proof. Let $u_{\varepsilon} \in V$ stand for the exact solution of (3). Owing to Theorem 2.4, $u_{\varepsilon}$ is bounded in $V$ so there is $u \in V$ such that $u_{\varepsilon} \rightarrow u$ in $V$. From (3) and since $\left|u_{\varepsilon}\right|_{H^{1}(\Omega)} \leq c\|f\|_{L^{2}(\Omega)}$, it follows using the Poincaré inequality in $V$, that

$$
b\left(u_{\varepsilon}, u_{\varepsilon}\right) \leq \varepsilon\left(f, u_{\varepsilon}\right) \lesssim \varepsilon\|f\|_{L^{2}(\Omega)}^{2},
$$

so that $b\left(u_{\varepsilon}, u_{\varepsilon}\right) \rightarrow 0$ when $\varepsilon \rightarrow 0$. Observing that $v \in V \mapsto b(v, v)$ is continuous from Lemma 2.3 and convex, it follows that $0 \leq b(u, u) \leq \liminf b\left(u_{\varepsilon}, u_{\varepsilon}\right)=0$. Then $u \in K$. Let us prove now that $u$ is the unique solution of (8). Let $w \in K$ and take $v=w-u_{\varepsilon}$ in (3). It then follows

$$
a\left(u_{\varepsilon}, w-u_{\varepsilon}\right)-\left(f, w-u_{\varepsilon}\right)=-\frac{1}{\varepsilon} b\left(u_{\varepsilon}, w-u_{\varepsilon}\right)=\frac{1}{\varepsilon}\left(b\left(w, w-u_{\varepsilon}\right)-b\left(u_{\varepsilon}, w-u_{\varepsilon}\right)\right),
$$

with $b\left(w, w-u_{\varepsilon}\right)=0$. Owing to the strong monotonicity of $b$, we then obtain $a\left(u_{\varepsilon}, w-\right.$ $\left.u_{\varepsilon}\right)-\left(f, w-u_{\varepsilon}\right) \geq 0$, so that

$$
a\left(u_{\varepsilon}, w\right)-\left(f, w-u_{\varepsilon}\right) \geq a\left(u_{\varepsilon}, u_{\varepsilon}\right)
$$

Since $\lim \inf a\left(u_{\varepsilon}, u_{\varepsilon}\right) \geq a(u, u)$, it then follows that $u \in K$ is the unique solution of (8). It now remains to prove that $u_{\varepsilon} \rightarrow u$ in $V$. This is readily obtained by proceeding similarly with $w=u$ so that $a\left(u_{\varepsilon}, u\right)-\left(f, u-u_{\varepsilon}\right) \geq a\left(u_{\varepsilon}, u_{\varepsilon}\right)$ and

$$
|u|_{H^{1}(\Omega)}^{2}=a(u, u) \geq \lim \sup \left|u_{\varepsilon}\right|_{H^{1}(\Omega)}^{2} .
$$

As a result $\lim \inf \left|u_{\varepsilon}\right|_{H^{1}(\Omega)} \geq|u|_{H^{1}(\Omega)} \geq \lim \sup \left|u_{\varepsilon}\right|_{H^{1}(\Omega)}$, so that $u_{\varepsilon} \rightarrow u$ in $V$.

\section{Finite element approximation}

In the rest of the paper, to lighten the notation we write $u$ instead of $u_{\varepsilon}$, so $u$ is the unique solution to the problem (3) which we now write as follows:

$$
u \in V \quad: \quad a(u, v)+\frac{1}{\varepsilon} b(u, v)=\int_{\Omega} f v, \quad \forall v \in V .
$$

We consider an approximation of the solution to this problem by standard Lagrange finite elements of first order. Consider $\mathcal{T}_{h}$ an affine mesh of $\Omega \subset \mathbb{R}^{d}$, with $d \in\{2,3\}$, regular in the sense of Ciarlet, composed of closed triangles $K \in \mathcal{T}_{h}$ and faces $F \in \mathcal{F}_{h}$. We denote by $h_{K}$ the diameter of an element $K$, and $h=\max _{K \in \mathcal{T}_{h}}$ represents the mesh size. Denoting 
by $h_{F}$ the size of a face $F \in \mathcal{F}_{h}$, mesh regularity yields that $h_{F} \sim h_{K}$ where $K$ stands for the triangle having $F$ on its boundary. In the forthcoming cases, when $d=2, \alpha>2$ or $d=3, \alpha=2$ (but not $d=2, \alpha=2$ ) we need to use inverse inequalites on $\Gamma_{C}$ so we have to suppose that the $d-1$ dimensional trace mesh on $\Gamma_{C}$ is quasi-uniform. The space $V$ is now approximated by the $\mathbb{P}_{1}$ Lagrange finite element space defined by

$$
V_{h}:=\left\{v_{h} \in V \mid \forall K \in \mathcal{T}_{h}, v_{h \mid K} \in \mathbb{P}_{1}(K)\right\} .
$$

The discrete problem issued from (9) is then

$$
u_{h} \in V_{h} \quad: \quad a\left(u_{h}, v_{h}\right)+\frac{1}{\varepsilon} b\left(u_{h}, v_{h}\right)=\int_{\Omega} f v_{h}, \quad \forall v_{h} \in V_{h} .
$$

This approximation being conformed, we immediately obtain from Theorem 2.4 the wellposedness of (10), and the discrete solution $u_{h}$ satisfies as well the a priori bound (7). We now examine the convergence of the solution $u_{h}$ to $u$.

Lemma 3.1 (A priori error estimate). Let $\alpha$ satisfy (5). Let $u \in V$ and $u_{h} \in V_{h}$ be the exact solutions of (9) and (10), respectively. Assume that $u \in W^{2, \alpha+1}(\Omega)$. Then,

$$
\left|u-u_{h}\right|_{H^{1}(\Omega)}^{2}+\frac{1}{\varepsilon}\left\|[u]_{+}-\left[u_{h}\right]_{+}\right\|_{L^{\alpha+1}\left(\Gamma_{C}\right)}^{\alpha+1} \lesssim h^{2}|u|_{H^{2}(\Omega)}^{2}+h^{2}\left(\frac{h}{\varepsilon}\right)^{\frac{1}{\alpha}}|u|_{W^{2, \alpha+1}(\Omega)}^{\frac{\alpha+1}{\alpha}}\|f\|_{L^{2}(\Omega)}^{2(\alpha-1) / \alpha} .
$$

Proof. Let $u \in V$ and $u_{h} \in V_{h}$ stand for the solutions of (9) and (10), respectively, and consider $\pi_{h}: W^{2, p}(\Omega) \rightarrow V_{h}$ the Lagrange interpolation operator in $V_{h}$, with $p>d / 2$. By definition, we have

$$
\left|u-u_{h}\right|_{H^{1}(\Omega)}^{2}=a\left(u-u_{h}, u-\pi_{h} u\right)+a\left(u-u_{h}, \pi_{h} u-u_{h}\right) .
$$

Applying Cauchy-Schwarz and Young inequalities, we obtain

$$
\frac{1}{2}\left|u-u_{h}\right|_{H^{1}(\Omega)}^{2} \leq \frac{1}{2}\left|\pi_{h} u-u\right|_{H^{1}(\Omega)}^{2}+a\left(u-u_{h}, \pi_{h} u-u_{h}\right) .
$$

By definition, we have

$$
\begin{aligned}
a\left(u-u_{h}, \pi_{h} u-u_{h}\right) & =\frac{1}{\varepsilon}\left(b\left(u_{h}, \pi_{h} u-u_{h}\right)-b\left(u, \pi_{h} u-u_{h}\right)\right) \\
& =-\frac{1}{\varepsilon} \int_{\Gamma_{C}}\left([u]_{+}^{\alpha}-\left[u_{h}\right]_{+}^{\alpha}\right)\left(\pi_{h} u-u_{h}\right)
\end{aligned}
$$

so that

$$
a\left(u-u_{h}, \pi_{h} u-u_{h}\right)=-\frac{1}{\varepsilon} \int_{\Gamma_{C}}\left([u]_{+}^{\alpha}-\left[u_{h}\right]_{+}^{\alpha}\right)\left(\pi_{h} u-u\right)-\frac{1}{\varepsilon} \int_{\Gamma_{C}}\left([u]_{+}^{\alpha}-\left[u_{h}\right]_{+}^{\alpha}\right)\left(u-u_{h}\right) .
$$

Applying Proposition 2.1, it then follows

$$
a\left(u-u_{h}, \pi_{h} u-u_{h}\right) \leq-\frac{1}{\varepsilon} \int_{\Gamma_{C}}\left([u]_{+}^{\alpha}-\left[u_{h}\right]_{+}^{\alpha}\right)\left(\pi_{h} u-u\right)-\frac{c}{\varepsilon}\left\|[u]_{+}-\left[u_{h}\right]_{+}\right\|_{L^{\alpha+1}\left(\Gamma_{C}\right)}^{\alpha+1},
$$


and then,

$\frac{1}{2}\left|u-u_{h}\right|_{H^{1}(\Omega)}^{2}+\frac{c}{\varepsilon}\left\|[u]_{+}-\left[u_{h}\right]_{+}\right\|_{L^{\alpha+1}\left(\Gamma_{C}\right)}^{\alpha+1} \leq \frac{1}{2}\left|\pi_{h} u-u\right|_{H^{1}(\Omega)}^{2}-\frac{1}{\varepsilon} \int_{\Gamma_{C}}\left([u]_{+}^{\alpha}-\left[u_{h}\right]_{+}^{\alpha}\right)\left(\pi_{h} u-u\right)$.

Finally, the last term is bounded using (6), to obtain

$$
\begin{aligned}
& \left|u-u_{h}\right|_{H^{1}(\Omega)}^{2}+\frac{1}{\varepsilon}\left\|[u]_{+}-\left[u_{h}\right]_{+}\right\|_{L^{\alpha+1}\left(\Gamma_{C}\right)}^{\alpha+1} \lesssim\left|\pi_{h} u-u\right|_{H^{1}(\Omega)}^{2} \\
& \quad+\frac{1}{\varepsilon}\left\|[u]_{+}-\left[u_{h}\right]_{+}\right\|_{L^{\alpha+1}\left(\Gamma_{C}\right)}\left\|\pi_{h} u-u\right\|_{L^{\alpha+1}\left(\Gamma_{C}\right)}\left(\left\|[u]_{+}\right\|_{L^{\alpha+1}\left(\Gamma_{C}\right)}^{\alpha-1}+\left\|\left[u_{h}\right]_{+}\right\|_{L^{\alpha+1}\left(\Gamma_{C}\right)}^{\alpha-1}\right) .
\end{aligned}
$$

Since $u$ and $u_{h}$ solve (9) and (10), respectively, the a priori bound (7) gives

$$
\varepsilon^{-\frac{\alpha-1}{\alpha+1}}\left(\left\|[u]_{+}\right\|_{L^{\alpha+1}\left(\Gamma_{C}\right)}^{\alpha-1}+\left\|\left[u_{h}\right]_{+}\right\|_{L^{\alpha+1}\left(\Gamma_{C}\right)}^{\alpha-1}\right) \lesssim\|f\|_{L^{2}(\Omega)}^{2(\alpha-1) /(\alpha+1)},
$$

so that

$$
\frac{1}{\varepsilon}\left(\left\|[u]_{+}\right\|_{L^{\alpha+1}\left(\Gamma_{C}\right)}^{\alpha-1}+\left\|\left[u_{h}\right]_{+}\right\|_{L^{\alpha+1}\left(\Gamma_{C}\right)}^{\alpha-1}\right) \lesssim \frac{1}{\varepsilon^{\frac{2}{\alpha+1}}}\|f\|_{L^{2}(\Omega)}^{2(\alpha-1) /(\alpha+1)}
$$

and we obtain

$$
\begin{aligned}
\left|u-u_{h}\right|_{H^{1}(\Omega)}^{2}+\frac{1}{\varepsilon}\left\|[u]_{+}-\left[u_{h}\right]_{+}\right\|_{L^{\alpha+1}\left(\Gamma_{C}\right)}^{\alpha+1} \lesssim\left|\pi_{h} u-u\right|_{H^{1}(\Omega)}^{2} & \\
& +\varepsilon^{-\frac{2}{\alpha+1}}\left\|[u]_{+}-\left[u_{h}\right]_{+}\right\|_{L^{\alpha+1}\left(\Gamma_{C}\right)}\left\|\pi_{h} u-u\right\|_{L^{\alpha+1}\left(\Gamma_{C}\right)}\|f\|_{L^{2}(\Omega)}^{2(\alpha-1) /(\alpha+1)} .
\end{aligned}
$$

Now, using the generalized Young inequality $a b \leq a^{p}+b^{q} /\left(q p^{\frac{q}{p}}\right)$ for all $a, b \geq 0$ and $p^{-1}+$ $q^{-1}=1$, it follows with $p=\alpha+1, \alpha q=\alpha+1, a=\varepsilon^{-\frac{1}{\alpha+1}}\left\|[u]_{+}-\left[u_{h}\right]_{+}\right\|_{L^{\alpha+1}\left(\Gamma_{C}\right)}, b=$ $\varepsilon^{-\frac{1}{\alpha+1}}\left\|\pi_{h} u-u\right\|_{L^{\alpha+1}\left(\Gamma_{C}\right)}\|f\|_{L^{2}(\Omega)}^{2(\alpha-1) /(\alpha+1)}$, that

$$
\left|u-u_{h}\right|_{H^{1}(\Omega)}^{2}+\frac{1}{\varepsilon}\left\|[u]_{+}-\left[u_{h}\right]_{+}\right\|_{L^{\alpha+1}\left(\Gamma_{C}\right)}^{\alpha+1} \lesssim\left|\pi_{h} u-u\right|_{H^{1}(\Omega)}^{2}+\varepsilon^{-\frac{1}{\alpha}}\left\|\pi_{h} u-u\right\|_{L^{\alpha+1}\left(\Gamma_{C}\right)}^{\frac{\alpha+1}{\alpha}}\|f\|_{L^{2}(\Omega) / \alpha}^{2(\alpha-1) / \alpha} .
$$

Finally, owing to Proposition 5.1 and using the regularity of $u$, we obtain

$$
\left|u-u_{h}\right|_{H^{1}(\Omega)}^{2}+\frac{1}{\varepsilon}\left\|[u]_{+}-\left[u_{h}\right]_{+}\right\|_{L^{\alpha+1}\left(\Gamma_{C}\right)}^{\alpha+1} \lesssim h^{2}|u|_{H^{2}(\Omega)}^{2}+h^{2+\frac{1}{\alpha}} \varepsilon^{-\frac{1}{\alpha}}|u|_{W^{2, \alpha+1}(\Omega)}^{\frac{\alpha+1}{\alpha}}\|f\|_{L^{2}(\Omega)}^{2(\alpha-1) / \alpha} .
$$

We immediately obtain the following corollary.

Corollary 3.2. Let $\alpha$ satisfy (5). Let $u \in V$ and $u_{h} \in V_{h}$ be the exact solutions of (9) and (10), respectively. Assume that $u \in W^{2, \alpha+1}(\Omega)$.

i) If $\varepsilon \gtrsim h$, then

$$
\left|u-u_{h}\right|_{H^{1}(\Omega)} \lesssim h\left(|u|_{H^{2}(\Omega)}^{2}+|u|_{W^{2, \alpha+1}(\Omega)}^{\frac{\alpha+1}{\alpha}}\|f\|_{L^{2}(\Omega)}^{2(\alpha-1) / \alpha}\right)^{\frac{1}{2}}
$$


ii) If $\varepsilon \sim h$, then

$$
\left|u-u_{h}\right|_{H^{1}(\Omega)}+h^{-\frac{1}{2}}\left\|[u]_{+}-\left[u_{h}\right]_{+}\right\|_{L^{\alpha+1}\left(\Gamma_{C}\right)}^{(\alpha+1) / 2} \lesssim h\left(|u|_{H^{2}(\Omega)}^{2}+|u|_{W^{2, \alpha+1}(\Omega)}^{\frac{\alpha+1}{\alpha}}\|f\|_{L^{2}(\Omega)}^{2(\alpha-1) / \alpha}\right)^{\frac{1}{2}} .
$$

Remark 3.3. When $\alpha=1$ and $d \in\{2,3\}$, the term $\|f\|_{L^{2}(\Omega)}$ disppears in the corollary, and we retrieve the estimates already obtained in [7] and more recently in [11] under the assumption $\varepsilon \sim h$. When $\alpha>1$, these estimates are new. In particular, the optimal $H^{1}$-convergence rate is obtained independently of $\alpha$ under the condition $\varepsilon \sim h$.

Remark 3.4. Let us briefly discuss on the regularity of problem (2). To our knowledge there does not exist any regularity results for problem (2). In order to have an idea concerning its regularity we consider a slightly different problem in which $\Gamma_{D}=\emptyset$ to avoid additional singularities at the interface between $\Gamma_{D}$ and $\Gamma_{C}$. Consider the problem of finding $u_{\varepsilon}$ s.t.

$$
\begin{aligned}
-\Delta u_{\varepsilon}+u_{\varepsilon}=f & \text { in } \Omega, \\
\varepsilon \partial_{n} u_{\varepsilon}+\left[u_{\varepsilon}\right]_{+}^{\alpha}=0 & \text { on } \partial \Omega,
\end{aligned}
$$

which admits a unique weak solution in $H^{1}(\Omega)$ so $u_{\varepsilon} \in H^{1 / 2}(\partial \Omega)$. Suppose that $\alpha=1$, so $\partial_{n} u_{\varepsilon}=-\frac{1}{\varepsilon}\left[u_{\varepsilon}\right]_{+} \in H^{1 / 2}(\partial \Omega)$ since $\left\|\left[u_{\varepsilon}\right]_{+}\right\|_{H^{1 / 2}(\partial \Omega)} \leq\left\|u_{\varepsilon}\right\|_{H^{1 / 2}(\partial \Omega)}$ and finally $u_{\varepsilon} \in W^{2,2}(\Omega)$. When $\alpha>1$, e.g. suppose $\alpha=2$ and $d=2$, assume that there is a small $\theta>0$ s.t. $u_{\varepsilon} \in H^{1+\theta}(\Omega)$. As before $\left[u_{\varepsilon}\right]_{+} \in H^{1 / 2+\theta}(\partial \Omega)$ and the latter space being a multiplicative algebra, we deduce that $\left[u_{\varepsilon}\right]_{+}^{2} \in H^{1 / 2+\theta}(\partial \Omega)$ so $\partial_{n} u_{\varepsilon} \in H^{1 / 2+\theta}(\partial \Omega)$, and then $u_{\varepsilon} \in W^{2+\theta, 2}(\Omega)$. Then, a bootstrap argument yields $u_{\varepsilon} \in H^{3 / 2+\theta}(\partial \Omega)$, so that $\forall \eta>0,\left[u_{\varepsilon}\right]_{+} \in H^{3 / 2-\eta}(\partial \Omega)$ (see [29]), and if $f$ is regular enough and $\eta$ small enough, $u_{\varepsilon} \in W^{3-\eta, 2}(\Omega) \hookrightarrow W^{2,3}(\Omega)$ by imbedding.

Remark 3.5. When $u$ is less regular than $W^{2, \alpha+1}(\Omega)$, say $u \in W^{s, \alpha+1}(\Omega), 1<s<2$ we can obtain a bound of $h^{2(s-1)}|u|_{H^{s}(\Omega)}^{2}+h^{2(s-1)}\left(\frac{h}{\varepsilon}\right)^{\frac{1}{\alpha}}|u|_{W^{s, \alpha+1}(\Omega)}^{\frac{\alpha+1}{\alpha}}\|f\|_{L^{2}(\Omega)}^{2(\alpha-1) / \alpha}$ in Lemma 3.1 and a bound of $C h^{s-1}$ in Corollary $3.2 i$ ) and $\left.i i\right)$. This is straightforward when $u$ is continuous which is always the case when $d=2$ or $d=3, s(\alpha+1)>3$. In the remaining cases, the Lagrange operator fails and interpolation operators adapted to nonsmooth functions should be considered.

\section{A priori error analysis in weak norm}

\subsection{The companion problem}

Let $u \in V$ and $u_{h} \in V_{h}$ be the exact and the approximated solutions of (9) and (10), respectively. Denote by $\zeta=u-u_{h} \in V$ the error, and assume that $\alpha$ satisfies (5) and $\alpha \neq 1$. Following the idea of [19] in a slightly different context, we introduce the companion problem

$$
\phi \in V \quad: \quad a(\phi, v)+\frac{1}{\varepsilon} \int_{\Gamma_{C}} q_{\alpha}^{\prime}(u) \phi v=\int_{\Omega} \zeta v, \quad \forall v \in V
$$

where $a$ is the bilinear map defined in $(4), q_{\alpha}(u)=[u]_{+}^{\alpha}$, so $q_{\alpha}^{\prime}(u)=\alpha[u]_{+}^{\alpha-1}$. 
As a linear problem with mixed Dirichlet-Robin boundary condition, problem (12) is well-posed and the solution $\phi \in V$ satisfies $|\phi|_{H^{1}(\Omega)} \lesssim\|\zeta\|_{L^{2}(\Omega)}$ uniformly in $\varepsilon$. Under some assumptions on $\Omega$ and the interface between $\Gamma_{C}$ and $\Gamma_{D}$ (see [26]), we know that the solution indeed belongs to $H^{2}(\Omega)$. In some particular cases (see [18, Theorem 4.3.1.4]), the solution $\phi \in V$ also satisifes the regularity estimate

$$
\|\phi\|_{H^{2}(\Omega)} \leq c\left(\varepsilon^{-1} q_{\alpha}^{\prime}(u)\right)\|\zeta\|_{L^{2}(\Omega)}
$$

with $c\left(\varepsilon^{-1} q_{\alpha}^{\prime}(u)\right)$ a constant that depends on its argument. In the following, we will assume that the following assumption holds.

Assumption 4.1. The unique solution $\phi \in V$ of (12) satisfies the stability estimate $|\phi|_{H^{2}(\Omega)} \leq$ $c\|\zeta\|_{L^{2}(\Omega)}$ with $c>0$ independent of $\varepsilon$ and $u$.

In what follows, we denote by $\phi_{h}=\pi_{h} \phi$ the Lagrange interpolation in $V_{h}$ of $\phi$. Usin assumption 4.1 and the inverse inequality, we obtain

$$
\left\|\phi-\phi_{h}\right\|_{L^{2}\left(\Gamma_{C}\right)}+h^{\frac{1}{2}}\left|\phi-\phi_{h}\right|_{H^{1}(\Omega)} \lesssim h^{\frac{3}{2}}|\phi|_{H^{2}(\Omega)} \lesssim h^{\frac{3}{2}}\|\zeta\|_{L^{2}(\Omega)}
$$

Using the continuous embedding $H^{2}(\Omega) \hookrightarrow H^{3 / 2}\left(\Gamma_{C}\right)$, we also have for $d \in\{2,3\}$ :

$$
\left\|\phi_{h}\right\|_{L^{\infty}\left(\Gamma_{C}\right)} \leq\|\phi\|_{L^{\infty}\left(\Gamma_{C}\right)} \lesssim\|\phi\|_{H^{3 / 2}\left(\Gamma_{C}\right)} \lesssim\|\phi\|_{H^{2}(\Omega)} \lesssim\|\zeta\|_{L^{2}(\Omega)}
$$

\section{2 $\quad L^{2}(\Omega)$-norm estimate for $d=\alpha=2$}

Lemma 4.2. Let $d=2$ and $\alpha=2$. Let $u \in V$ be the unique solution of (9) and $u_{h} \in V_{h}$ the unique solution of (10), respectively. Assume that $u \in W^{2,3}(\Omega)$ and that $\varepsilon \geq \theta h$ with $\theta$ large enough. Then

$$
\left\|u-u_{h}\right\|_{L^{2}(\Omega)} \lesssim c\left(|u|_{W^{2,3}(\Omega)},\|f\|_{L^{2}(\Omega)}\right) h^{2},
$$

where $c\left(|u|_{W^{2,3}(\Omega)},\|f\|_{L^{2}(\Omega)}\right)$ denotes a constant that depends on its arguments.

Proof. Proceeding as for linear problems, we choose $v=\zeta$ in (12) to obtain

$$
\|\zeta\|_{L^{2}(\Omega)}^{2}=a(\phi, \zeta)+\frac{1}{\varepsilon} \int_{\Gamma_{C}} q_{\alpha}^{\prime}(u) \phi \zeta .
$$

Denoting $\phi_{h}=\pi_{h} \phi$, using the definition of $u$ and $u_{h}$ and the Galerkin orthogonality, we infer from (9) and (10) that

$$
0=a\left(u, \phi_{h}\right)-a\left(u_{h}, \phi_{h}\right)+\frac{1}{\varepsilon} \int_{\Gamma_{C}}\left(q_{\alpha}(u)-q_{\alpha}\left(u_{h}\right)\right) \phi_{h}=a\left(\zeta, \phi_{h}\right)+\frac{1}{\varepsilon} \int_{\Gamma_{C}}\left(q_{\alpha}(u)-q_{\alpha}\left(u_{h}\right)\right) \phi_{h} .
$$

Subtracting the two previous equalities yields

$$
\|\zeta\|_{L^{2}(\Omega)}^{2}=a\left(\zeta, \phi-\phi_{h}\right)-\frac{1}{\varepsilon} \int_{\Gamma_{C}}\left(q_{\alpha}(u)-q_{\alpha}\left(u_{h}\right)\right) \phi_{h}+\frac{1}{\varepsilon} \int_{\Gamma_{C}} q_{\alpha}^{\prime}(u) \zeta \phi,
$$


or equivalently,

$$
\|\zeta\|_{L^{2}(\Omega)}^{2}=a\left(\zeta, \phi-\phi_{h}\right)-\frac{1}{\varepsilon} \int_{\Gamma_{C}}\left(q_{\alpha}(u)-q_{\alpha}\left(u_{h}\right)-q_{\alpha}^{\prime}(u) \zeta\right) \phi_{h}+\frac{1}{\varepsilon} \int_{\Gamma_{C}} q_{\alpha}^{\prime}(u) \zeta\left(\phi-\phi_{h}\right) .
$$

We then write $\|\zeta\|_{L^{2}(\Omega)}^{2}=T_{1}+T_{2}+T_{3}$ with

$$
\begin{aligned}
T_{1} & =a\left(\zeta, \phi-\phi_{h}\right), \\
T_{2} & =-\frac{1}{\varepsilon} \int_{\Gamma_{C}}\left(q_{\alpha}(u)-q_{\alpha}\left(u_{h}\right)-q_{\alpha}^{\prime}(u) \zeta\right) \phi_{h}, \\
T_{3} & =\frac{1}{\varepsilon} \int_{\Gamma_{C}} q_{\alpha}^{\prime}(u) \zeta\left(\phi-\phi_{h}\right) .
\end{aligned}
$$

To obtain an estimate of $\|\zeta\|_{L^{2}(\Omega)}$, we consider separately each term $T_{1}, T_{2}$ and $T_{3}$. For $T_{1}$, we apply Cauchy-Schwarz inequality with (13) and Corollary 3.2 to obtain

$$
\left|T_{1}\right| \leq\left|u-u_{h}\right|_{H^{1}(\Omega)}\left|\phi-\phi_{h}\right|_{H^{1}(\Omega)} \lesssim c(u, f)^{\frac{1}{2}} h^{2}\|\zeta\|_{L^{2}(\Omega)},
$$

with $c(u, f)=\left(|u|_{H^{2}(\Omega)}^{2}+|u|_{W^{2,3}(\Omega)}^{3 / 2}\|f\|_{L^{2}(\Omega)}\right)$. Considering now the second term $T_{2}$, we observe that

$$
q_{\alpha}(u)-q_{\alpha}\left(u_{h}\right)-q_{\alpha}^{\prime}(u) \zeta=\zeta \int_{0}^{1}\left(q_{\alpha}^{\prime}(u-t \zeta)-q_{\alpha}^{\prime}(u)\right) d t
$$

so that $T_{2}$ can be written as

$$
T_{2}=-\frac{1}{\varepsilon} \int_{\Gamma_{C}} \zeta \phi_{h}\left(\int_{0}^{1}\left(q_{\alpha}^{\prime}(u-t \zeta)-q_{\alpha}^{\prime}(u)\right) d t\right) .
$$

To estimate the integral over $t$, we note that $t \in \mathbb{R} \mapsto[t]_{+}$is 1 -Lipschitz, so that in particular

$$
\int_{0}^{1}\left|q_{\alpha}^{\prime}(u-t \zeta)-q_{\alpha}^{\prime}(u)\right| d t \lesssim|\zeta|
$$

Then, the simple estimate follows:

$$
\left|T_{2}\right| \lesssim \frac{1}{\varepsilon} \int_{\Gamma_{C}}|\zeta|^{2}\left|\phi_{h}\right|
$$

Applying Holder's inequality combined with the trace inequality $\|\zeta\|_{L^{2}\left(\Gamma_{C}\right)} \leq\|\zeta\|_{L^{2}(\Omega)}^{1 / 2}|\zeta|_{H^{1}(\Omega)}^{1 / 2}$, estimate (14) and Corollary 3.2, we obtain

$$
\left|T_{2}\right| \lesssim \frac{1}{\varepsilon}\|\zeta\|_{L^{2}\left(\Gamma_{C}\right)}^{2}\left\|\phi_{h}\right\|_{L^{\infty}\left(\Gamma_{C}\right)} \lesssim \frac{1}{\varepsilon}\|\zeta\|_{L^{2}(\Omega)}^{2}|\zeta|_{H^{1}(\Omega)} \lesssim c(u, f)^{\frac{1}{2}} \frac{h}{\varepsilon}\|\zeta\|_{L^{2}(\Omega)}^{2} .
$$

For the last term $T_{3}$, we have

$$
T_{3}=\frac{2}{\varepsilon} \int_{\Gamma_{C}}[u]_{+} \zeta\left(\phi-\phi_{h}\right)
$$


Then, applying Holder's inequality with $\frac{1}{3}+\frac{1}{2}+\frac{1}{6}=1$ provides

$$
\left|T_{3}\right| \lesssim \frac{1}{\varepsilon}\left\|[u]_{+}\right\|_{L^{3}\left(\Gamma_{C}\right)}\|\zeta\|_{L^{2}\left(\Gamma_{C}\right)}\left\|\phi-\phi_{h}\right\|_{L^{6}\left(\Gamma_{C}\right)}
$$

From (7), we know that $\left\|[u]_{+}\right\|_{L^{3}\left(\Gamma_{C}\right)} \lesssim \varepsilon^{\frac{1}{3}}\|f\|_{L^{2}(\Omega)}^{2 / 3}$. Then, using the trace inequality $\|\zeta\|_{L^{2}\left(\Gamma_{C}\right)} \leq$ $\|\zeta\|_{L^{2}(\Omega)}^{1 / 2}|\zeta|_{H^{1}(\Omega)}^{1 / 2}$, we deduce from Corollary 3.2 that

$$
\left|T_{3}\right| \lesssim\|f\|_{L^{2}(\Omega)}^{2 / 3} \frac{1}{\varepsilon^{2 / 3}}\|\zeta\|_{L^{2}(\Omega)}^{1 / 2} h^{1 / 2} c(u, f)^{1 / 4}\left\|\phi-\phi_{h}\right\|_{L^{6}\left(\Gamma_{C}\right)}
$$

It now remains to estimate $\left\|\phi-\phi_{h}\right\|_{L^{6}\left(\Gamma_{C}\right)}$. Observing that the embedding $W^{2,2}(\Omega) \rightarrow$ $W^{\frac{4}{3}, 6}(\Omega)$ is continuous (see [1]), we infer from Proposition 5.1 that

$$
\left\|\phi-\phi_{h}\right\|_{L^{6}\left(\Gamma_{C}\right)} \lesssim h^{\frac{4}{3}-\frac{1}{6}}|\phi|_{W^{\frac{4}{3}, 6}(\Omega)} \lesssim h^{\frac{7}{6}}|\phi|_{H^{2}(\Omega)} \lesssim h^{\frac{7}{6}}\|\zeta\|_{L^{2}(\Omega)}
$$

Hence, for $T_{3}$ it follows

$$
\left|T_{3}\right| \lesssim\left(\frac{h}{\varepsilon}\right)^{2 / 3} h\|\zeta\|_{L^{2}(\Omega)}^{3 / 2}\|f\|_{L^{2}(\Omega)}^{2 / 3} c(u, f)^{1 / 4} .
$$

Collecting the estimates on $T_{1}, T_{2}$ and $T_{3}$, and absorbing the constants in the $\lesssim$ symbol, it then follows

$$
\|\zeta\|_{L^{2}(\Omega)}^{2} \lesssim\left(h^{2}+\frac{h}{\varepsilon}\|\zeta\|_{L^{2}(\Omega)}\right)\|\zeta\|_{L^{2}(\Omega)}+\left(\frac{h}{\varepsilon}\right)^{2 / 3} h\|\zeta\|_{L^{2}(\Omega)}^{3 / 2} .
$$

We then use the hypothesis $\varepsilon \geq \theta h$ to infer

$$
\|\zeta\|_{L^{2}(\Omega)} \lesssim h^{2}+\theta^{-1}\|\zeta\|_{L^{2}(\Omega)}+\theta^{-2 / 3} h\|\zeta\|_{L^{2}(\Omega)}^{1 / 2} .
$$

Since $\theta$ is assumed large enough, the expected result follows from the previous bound using Young's inequality.

Remark 4.3. When $u$ is less regular than $W^{2,3}(\Omega)$, say $u \in W^{s, 3}(\Omega), 1<s<2$ we can check that the limiting term is $T_{2}$ which requires that $\varepsilon=\theta h^{s-1}$; we then obtain an $L^{2}$-error bound of $h^{s}$ in Lemma 4.2.

\section{3 $L^{2}(\Omega)$-norm estimate for $d=3$ and $\alpha=2$}

In this section, we extend the previous analysis to a three dimensional domain $\Omega$.

Lemma 4.4. Let $d=3$ and $\alpha=2$. Let $u \in V$ be the unique solution of (9) and $u_{h} \in V_{h}$ the unique solution of (10), respectively. Assume that $u \in W^{2,3}(\Omega)$ and that $\varepsilon \geq \theta$ with $\theta$ large enough. Then

$$
\left\|u-u_{h}\right\|_{L^{2}(\Omega)} \lesssim c\left(|u|_{W^{2,3}(\Omega)},\|f\|_{L^{2}(\Omega)}\right) h^{\frac{8}{5}}
$$

If in addition $\varepsilon \geq \theta \sqrt{h}$,

$$
\left\|u-u_{h}\right\|_{L^{2}(\Omega)} \lesssim c\left(|u|_{W^{2,3}(\Omega)},\|f\|_{L^{2}(\Omega)}\right) h^{2} .
$$


Proof. Proceeding as in the proof for $d=2$, we observe that (15) and (16) also hold for $d=3$. For the third term $T_{3}$ we apply Holder's inequality with $\frac{1}{3}+\frac{1}{2}+\frac{1}{6}=1$. Note that we choose $L^{6}$ for $\zeta$ and $L^{2}$ for $\phi-\phi_{h}$ which is the contrary of the choice for $d=2$. That choice gives the best estimates we are able to obtain in our analysis (we skip over the discussion on the choice which is technical). Hence, we obtain

$$
T_{3}=\frac{2}{\varepsilon} \int_{\Gamma_{C}}[u]_{+} \zeta\left(\phi-\phi_{h}\right) \leq \frac{2}{\varepsilon}\left\|[u]_{+}\right\|_{L^{3}\left(\Gamma_{C}\right)}\|\zeta\|_{L^{6}\left(\Gamma_{C}\right)}\left\|\phi-\phi_{h}\right\|_{L^{2}\left(\Gamma_{C}\right)}
$$

Using estimate (7), it then follows

$$
T_{3} \lesssim \frac{2}{\varepsilon^{2 / 3}}\|f\|_{L^{2}(\Omega)}^{2 / 3}\|\zeta\|_{L^{6}\left(\Gamma_{C}\right)}\left\|\phi-\phi_{h}\right\|_{L^{2}\left(\Gamma_{C}\right)}
$$

and using (13), we have $\left\|\phi-\phi_{h}\right\|_{L^{2}\left(\Gamma_{C}\right)} \lesssim h^{\frac{3}{2}}\|\zeta\|_{L^{2}(\Omega)}$. As a partial result, we obtain

$$
T_{3} \lesssim \frac{h^{\frac{3}{2}}}{\varepsilon^{\frac{2}{3}}}\|f\|_{L^{2}(\Omega)}^{2 / 3}\|\zeta\|_{L^{6}\left(\Gamma_{C}\right)}\|\zeta\|_{L^{2}(\Omega)} .
$$

Now, we combine the interpolation inequality $\|\zeta\|_{L^{6}\left(\Gamma_{C}\right)} \lesssim\|\zeta\|_{L^{2}\left(\Gamma_{C}\right)}^{1 / 3}\|\zeta\|_{H^{1}\left(\Gamma_{C}\right)}^{2 / 3}$ valid in two space dimension (see [3]), with the trace inequality $\|\zeta\|_{L^{2}\left(\Gamma_{C}\right)} \leq\|\zeta\|_{L^{2}(\Omega)}^{1 / 2}|\zeta|_{H^{1}(\Omega)}^{1 / 2}$ to obtain

$$
\|\zeta\|_{L^{6}\left(\Gamma_{C}\right)} \lesssim\|\zeta\|_{L^{2}(\Omega)}^{1 / 6}|\zeta|_{H^{1}(\Omega)}^{1 / 6}\|\zeta\|_{H^{1}\left(\Gamma_{C}\right)}^{2 / 3}
$$

We now estimate $\|\zeta\|_{H^{1}\left(\Gamma_{C}\right)}$ as follows:

$$
\begin{aligned}
\|\zeta\|_{H^{1}\left(\Gamma_{C}\right)} & \leq\left\|u-\pi_{h} u\right\|_{H^{1}\left(\Gamma_{C}\right)}+\left\|\pi_{h} u-u_{h}\right\|_{H^{1}\left(\Gamma_{C}\right)} \\
& \lesssim h^{1 / 2}\|u\|_{H^{3 / 2}\left(\Gamma_{C}\right)}+h^{-1 / 2}\left\|\pi_{h} u-u_{h}\right\|_{H^{1 / 2}\left(\Gamma_{C}\right)} \\
& \lesssim h^{1 / 2}\|u\|_{H^{2}(\Omega)}+h^{-1 / 2}\left\|u-u_{h}\right\|_{H^{1 / 2}\left(\Gamma_{C}\right)}+h^{-1 / 2}\left\|u-\pi_{h} u\right\|_{H^{1 / 2}\left(\Gamma_{C}\right)} \\
& \lesssim h^{1 / 2}\|u\|_{H^{2}(\Omega)}+h^{-1 / 2}|\zeta|_{H^{1}(\Omega)},
\end{aligned}
$$

where we have used triangular, interpolation, continuous and inverse inequalities, combined with the a priori estimate from Corollary 3.2. Then, we obtain

$$
\|\zeta\|_{L^{6}\left(\Gamma_{C}\right)} \lesssim\|\zeta\|_{L^{2}(\Omega)}^{1 / 6}|\zeta|_{H^{1}(\Omega)}^{1 / 6}\left(h^{1 / 3}\|u\|_{H^{2}(\Omega)}^{2 / 3}+h^{-1 / 3}|\zeta|_{H^{1}(\Omega)}^{2 / 3}\right) .
$$

From Corollary 3.2, it follows that $|\zeta|_{H^{1}(\Omega)} \leq c(u, f) h$ if $\varepsilon \geq \theta h$, so that

$$
\|\zeta\|_{L^{6}\left(\Gamma_{C}\right)} \lesssim c(u, f) h^{1 / 2}\|\zeta\|_{L^{2}(\Omega)}^{1 / 6}
$$

where $c(u, f)$ is some constant depending on $|u|_{W^{2,3}(\Omega)},\|u\|_{H^{2}(\Omega)}$ and $\|f\|_{L^{2}(\Omega)}$. Hence, for $T_{3}$ we obtain the estimate

$$
T_{3} \lesssim\left(\frac{h}{\varepsilon}\right)^{2 / 3} h^{4 / 3}\|\zeta\|_{L^{2}(\Omega)}^{7 / 6}
$$


which gives $T_{3} \lesssim \theta^{-2 / 3} h^{4 / 3}\|\zeta\|_{L^{2}(\Omega)}^{7 / 6}$ with $\varepsilon \geq \theta h$ bounded. Hence, applying generalized Young's inequality yields

$$
T_{3} \lesssim \theta^{-8 / 7}\|\zeta\|_{L^{2}(\Omega)}^{2}+h^{16 / 5}
$$

and since $\theta$ is large enough we get (18). If we consider again (20) with the additional constraint $\varepsilon \geq \theta h^{1 / 2}$, then

$$
T_{3} \lesssim\left(\frac{h^{1 / 2}}{\varepsilon}\right)^{2 / 3} h^{1 / 3} h^{4 / 3}\|\zeta\|_{L^{2}(\Omega)}^{7 / 6} \lesssim \theta^{-2 / 3} h^{5 / 3}\|\zeta\|_{L^{2}(\Omega)}^{7 / 6}
$$

which implies $T_{3} \lesssim \theta^{-8 / 7}\|\zeta\|_{L^{2}(\Omega)}^{2}+h^{4}$ and (19).

\subsection{Estimate in $L^{2}(\Omega)$-norm for $d=2, \alpha>2$}

Lemma 4.5. Let $d=2$ and $\alpha>2$. Let $u \in V$ be the unique solution of (9) and $u_{h} \in V_{h}$ the unique solution of (10). Assume that $u \in W^{2, \alpha+1}(\Omega), h \lesssim \varepsilon$ and $h$ small enough. Then

$$
\left\|u-u_{h}\right\|_{L^{2}(\Omega)} \leq c\left(\alpha,|u|_{W^{2, \alpha+1}(\Omega)},\|f\|_{L^{2}(\Omega)}\right) h^{2}(-\ln (h)),
$$

where $c\left(\alpha,|u|_{W^{2, \alpha+1}(\Omega)},\|f\|_{L^{2}(\Omega)}\right)$ is a constant that depends on its arguments.

Proof. As in the case $\alpha=2$ we consider separately each term $T_{1}, T_{2}$ and $T_{3}$ and we absorb the constants in the $\lesssim$ symbol. For $T_{1}$, we obtain as before

$$
\left|T_{1}\right| \leq\left|u-u_{h}\right|_{H^{1}(\Omega)}\left|\phi-\phi_{h}\right|_{H^{1}(\Omega)} \lesssim h^{2} c(u, f)^{1 / 2}\|\zeta\|_{L^{2}(\Omega)} \lesssim h^{2}\|\zeta\|_{L^{2}(\Omega)} .
$$

Considering now the second term $T_{2}$, we still have

$$
T_{2}=-\frac{1}{\varepsilon} \int_{\Gamma_{C}} \zeta \phi_{h}\left(\int_{0}^{1}\left(q_{\alpha}^{\prime}(u-t \zeta)-q_{\alpha}^{\prime}(u)\right) d t\right)=-\frac{\alpha}{\varepsilon} \int_{\Gamma_{C}} \zeta \phi_{h}\left(\int_{0}^{1}\left([u-t \zeta]_{+}^{\alpha-1}-[u]_{+}^{\alpha-1}\right) d t\right) .
$$

The estimate on the integral over $t$ is now different, and we use the bounds of Lemma 2.3:

$$
\int_{0}^{1}\left([u-t \zeta]_{+}^{\alpha-1}-[u]_{+}^{\alpha-1}\right) d t \lesssim \int_{0}^{1}\left([u-t \zeta]_{+}-[u]_{+}\right)\left([u-t \zeta]_{+}^{\alpha-2}+[u]_{+}^{\alpha-2}\right) d t
$$

Since $[u-t \zeta]_{+} \leq[u]_{+}+\left[u_{h}\right]_{+}$, we obtain $[u-t \zeta]_{+}^{\alpha-2}+[u]_{+}^{\alpha-2} \leq C\left([u]_{+}^{\alpha-2}+\left[u_{h}\right]_{+}^{\alpha-2}\right)$ and then

$$
\int_{0}^{1}\left([u-t \zeta]_{+}^{\alpha-1}-[u]_{+}^{\alpha-1}\right) d t \lesssim|\zeta|\left([u]_{+}^{\alpha-2}+\left[u_{h}\right]_{+}^{\alpha-2}\right) .
$$

It follows the estimate

$$
\begin{aligned}
\left|T_{2}\right| & \lesssim \frac{\alpha}{\varepsilon} \int_{\Gamma_{C}} \zeta^{2}\left|\phi_{h}\right|\left([u]_{+}^{\alpha-2}+\left[u_{h}\right]_{+}^{\alpha-2}\right) \\
& \lesssim \frac{\alpha}{\varepsilon}\left\|\zeta^{2}\right\|_{L^{\frac{\alpha+1}{3}}\left(\Gamma_{C}\right)}\left\|\phi_{h}\right\|_{L^{\infty}\left(\Gamma_{C}\right)}\left(\left\|[u]_{+}\right\|_{L^{\alpha+1}\left(\Gamma_{C}\right)}^{\alpha-2}+\left\|\left[u_{h}\right]_{+}\right\|_{L^{\alpha+1}\left(\Gamma_{C}\right)}^{\alpha-2}\right)
\end{aligned}
$$


where Holder's inequality is used with $\frac{3}{\alpha+1}+\frac{\alpha-2}{\alpha+1}=1$. Using the a priori bound (7) for $u$ and $u_{h}$, it implies

$$
\begin{aligned}
& \left|T_{2}\right| \lesssim \frac{\alpha}{\varepsilon}\left\|\zeta^{2}\right\|_{L^{\frac{\alpha+1}{3}}\left(\Gamma_{C}\right)}\left\|\phi_{h}\right\|_{L^{\infty}\left(\Gamma_{C}\right)^{\frac{\alpha-2}{\alpha+1}}\|f\|_{L^{2}(\Omega)}^{2(\alpha-2) /(\alpha+1)}} \\
& \lesssim \alpha\|\zeta\|_{L^{\frac{2(\alpha+1)}{3}}\left(\Gamma_{C}\right)}^{2}\|\zeta\|_{L^{2}(\Omega)} \varepsilon^{\frac{-3}{\alpha+1}}\|f\|_{L^{2}(\Omega)}^{2(\alpha-2) /(\alpha+1)},
\end{aligned}
$$

where we have used (14) to infer that $\left\|\phi_{h}\right\|_{L^{\infty}\left(\Gamma_{C}\right)} \lesssim\|\zeta\|_{L^{2}(\Omega)}$. It now remains to estimate $\|\zeta\|_{L^{\frac{2(\alpha+1)}{3}}\left(\Gamma_{C}\right)}^{2}$. Using the interpolation inequality $\|\zeta\|_{L^{\frac{2(\alpha+1)}{3}}\left(\Gamma_{C}\right)} \leq\|\zeta\|_{L^{2}\left(\Gamma_{C}\right)}^{\frac{3}{\alpha+1}}\|\zeta\|_{L^{\infty}\left(\Gamma_{C}\right)}^{\frac{\alpha-2}{\alpha+1}}$ combined with the trace inequality $\|\zeta\|_{L^{2}\left(\Gamma_{C}\right)} \lesssim\|\zeta\|_{L^{2}(\Omega)}^{1 / 2}|\zeta|_{H^{1}(\Omega)}^{1 / 2}$, we obtain

$$
\|\zeta\|_{L^{\frac{2(\alpha+1)}{3}\left(\Gamma_{C}\right)}} \lesssim\|\zeta\|_{L^{2}(\Omega)}^{\frac{3}{2(\alpha+1)}}|\zeta|_{H^{1}(\Omega)}^{\frac{3}{2(\alpha+1)}}\|\zeta\|_{L^{\infty}\left(\Gamma_{C}\right)}^{\frac{\alpha-2}{\alpha+1}}
$$

Then, using Corollary 3.2, it implies that

$$
\left|T_{2}\right| \lesssim \alpha\left(\frac{h}{\varepsilon}\right)^{\frac{3}{\alpha+1}}\|\zeta\|_{L^{2}(\Omega)}^{\frac{3}{\alpha+1}+1}\|\zeta\|_{L^{\infty}\left(\Gamma_{C}\right)}^{\frac{2(\alpha-2)}{\alpha+1}}
$$

Finally, we estimate $\|\zeta\|_{L^{\infty}\left(\Gamma_{C}\right)}$ using Proposition 5.2 and Corollary 3.2 as $\|\zeta\|_{L^{\infty}\left(\Gamma_{C}\right)} \lesssim$ $h \sqrt{-\ln (h)}$ yielding

$$
\left|T_{2}\right| \lesssim \alpha\left(\frac{h}{\varepsilon}\right)^{\frac{3}{\alpha+1}} h^{\frac{2(\alpha-2)}{\alpha+1}}(\sqrt{-\ln (h)})^{\frac{2(\alpha-2)}{\alpha+1}}\|\zeta\|_{L^{2}(\Omega)}^{\frac{3}{\alpha+1}+1} .
$$

Let us now consider the last term

$$
T_{3}=\frac{\alpha}{\varepsilon} \int_{\Gamma_{C}}[u]_{+}^{\alpha-1} \zeta\left(\phi-\phi_{h}\right)
$$

and apply Holder's inequality with $\frac{\alpha-1}{\alpha+1}+\frac{3}{2(\alpha+1)}+\frac{1}{2(\alpha+1)}=1$ to obtain

$$
\left|T_{3}\right| \leq \frac{\alpha}{\varepsilon}\left\|[u]_{+}\right\|_{L^{\alpha+1}\left(\Gamma_{C}\right)}^{\alpha-1}\|\zeta\|_{L^{\frac{2(\alpha+1)}{3}\left(\Gamma_{C}\right)}}\left\|\phi-\phi_{h}\right\|_{L^{2(\alpha+1)}\left(\Gamma_{C}\right)}
$$

Proceeding as above, we use the estimate $\left\|[u]_{+}\right\|_{L^{\alpha+1}\left(\Gamma_{C}\right)} \lesssim \varepsilon^{\frac{1}{\alpha+1}}\|f\|_{L^{2}(\Omega)}^{2 /(\alpha+1)}$ and the estimate $(23)$ on $\|\zeta\|_{L^{\frac{2(\alpha+1)}{3}}\left(\Gamma_{C}\right)}$ to obtain

$$
\left|T_{3}\right| \lesssim \frac{\alpha}{\varepsilon^{\frac{2}{\alpha+1}}}\|\zeta\|_{L^{2}(\Omega)}^{\frac{3}{2(\alpha+1)}}|\zeta|_{H^{1}(\Omega)}^{\frac{3}{2(\alpha+1)}}\|\zeta\|_{L^{\infty}\left(\Gamma_{C}\right)}^{\frac{\alpha-2}{\alpha+1}}\left\|\phi-\phi_{h}\right\|_{L^{2(\alpha+1)}\left(\Gamma_{C}\right)}
$$

Then, using Proposition 5.2 and Corollary 3.2, it follows

$$
\left|T_{3}\right| \lesssim \frac{\alpha}{\varepsilon^{\frac{2}{\alpha+1}}}\|\zeta\|_{L^{2}(\Omega)}^{\frac{3}{2(\alpha+1)}} h^{\frac{2 \alpha-1}{2(\alpha+1)}}(\sqrt{-\ln (h)})^{\frac{\alpha-2}{\alpha+1}}\left\|\phi-\phi_{h}\right\|_{L^{2(\alpha+1)}\left(\Gamma_{C}\right)} .
$$


Now, we use Proposition 5.1 with the continuous embedding $W^{2,2}(\Omega) \hookrightarrow W^{\frac{\alpha+2}{\alpha+1}, 2(\alpha+1)}(\Omega)$ to obtain

$$
\left\|\phi-\phi_{h}\right\|_{L^{2(\alpha+1)}\left(\Gamma_{C}\right)} \lesssim h^{\frac{\alpha+2}{\alpha+1}-\frac{1}{2(\alpha+1)}}|\phi|_{W^{\frac{\alpha+2}{\alpha+1}, 2(\alpha+1)}(\Omega)} \lesssim h^{\frac{2 \alpha+3}{2(\alpha+1)}}|\phi|_{W^{2,2}(\Omega)} \lesssim h^{\frac{2 \alpha+3}{2(\alpha+1)}}\|\zeta\|_{L^{2}(\Omega)} .
$$

Hence, we obtain

$$
\left|T_{3}\right| \lesssim \alpha\left(\frac{h}{\varepsilon}\right)^{\frac{2}{\alpha+1}} h^{\frac{4 \alpha-2}{2(\alpha+1)}}(\sqrt{-\ln (h)})^{\frac{\alpha-2}{\alpha+1}}\|\zeta\|_{L^{2}(\Omega)}^{\frac{3}{2(\Omega+1)}+1}
$$

Now, collecting the three estimates of $T_{1}, T_{2}, T_{3}$, it follows

$$
\begin{array}{r}
\|\zeta\|_{L^{2}(\Omega) \lesssim} \lesssim h^{2}+\left(\frac{h}{\varepsilon}\right)^{\frac{3}{\alpha+1}} h^{\frac{2(\alpha-2)}{\alpha+1}}\|\zeta\|_{L^{2}(\Omega)}^{\frac{3}{\alpha+1}}(\sqrt{-\ln (h)})^{\frac{2(\alpha-2)}{\alpha+1}}+ \\
\left(\frac{h}{\varepsilon}\right)^{\frac{2}{\alpha+1}} h^{\frac{2 \alpha-1}{\alpha+1}}\|\zeta\|_{L^{2}(\Omega)}^{\frac{3}{2(\alpha+1)}}(\sqrt{-\ln (h)})^{\frac{\alpha-2}{\alpha+1}},
\end{array}
$$

so that we conclude applying Young's inequality with the assumption $h \lesssim \varepsilon$.

Remark 4.6. Note that the estimate (17) obtained for $\alpha=2$ is consistent with the general estimate (24) valid for all $\alpha>2$. In addition, observe that the assumption $\varepsilon \geq \theta h$ with $\theta$ large enough is not needed if $\alpha>2$, since we have $\frac{3}{\alpha+1}<1$. Nevertheless a $|\ln (h)|$ term appears when $\alpha>2$.

\section{$5 \quad$ Technical results}

Let $\pi_{h}$ be the linear Lagrange interpolation operator which to any function $v \in W^{s, p}(\Omega)$ with $s p>d$ and vanishing on $\overline{\Gamma_{D}}$ associates its interpolant $\pi_{h} v \in V_{h}$. We recall the following properties.

Proposition 5.1. For all $v \in W^{2, p}(\Omega)$ with $2 p>d$,

$$
\begin{aligned}
& \left\|\pi_{h} v-v\right\|_{L^{p}(\Omega)}+h\left|\pi_{h} v-v\right|_{W^{1, p}(\Omega)} \lesssim h^{2}|v|_{W^{2, p}(\Omega)}, \\
& \left\|\pi_{h} v-v\right\|_{L^{p}\left(\Gamma_{C}\right)} \lesssim h^{2-\frac{1}{p}}|v|_{W^{2, p}(\Omega)} .
\end{aligned}
$$

More generally, if $v \in W^{s, p}(\Omega)$ with $s p>d$ and $1<s \leq 2$,

$$
\left\|\pi_{h} v-v\right\|_{L^{p}\left(\Gamma_{C}\right)} \lesssim h^{s-\frac{1}{p}}|v|_{W^{s, p}(\Omega)}
$$

Proof. For all $K \in \mathcal{T}_{h}$, the local interpolation theory gives us

$$
\left\|\pi_{h} v-v\right\|_{L^{p}(K)}+h_{K}\left|\pi_{h} v-v\right|_{W^{1, p}(K)} \lesssim h_{K}^{2}|v|_{W^{2, p}(K)} .
$$

Considering a boundary face $F$ touching the cell $K \in \Omega_{h}$, we have from the multiplicative trace inequality in $L^{p}(F)$ :

$$
\left\|\pi_{h} v-v\right\|_{L^{p}(F)} \lesssim\left\|\pi_{h} v-v\right\|_{L^{p}(K)}^{\frac{p-1}{p}}\left(h_{K}^{-\frac{1}{p}}\left\|\pi_{h} v-v\right\|_{L^{p}(K)}^{\frac{1}{p}}+\left\|\nabla\left(\pi_{h} v-v\right)\right\|_{L^{p}(K)}^{\frac{1}{p}}\right) .
$$


Hence, combining these two estimates, it follows

$$
\left\|\pi_{h} v-v\right\|_{L^{p}(F)} \lesssim h_{K}^{2-\frac{1}{p}}|v|_{W^{2, p}(K)}
$$

and we obtain, summing over $F \subset \Gamma_{C}$,

$$
\left\|\pi_{h} v-v\right\|_{L^{p}\left(\Gamma_{C}\right)}^{p} \leq \sum_{F \subset \Gamma_{C}} h_{K_{F}}^{2 p-1}|v|_{W^{2, p}\left(K_{F}\right)}^{p} \lesssim h^{2 p-1}|v|_{W^{2, p}(\Omega)}^{p} .
$$

The generalization to $1<s \leq 2$ follows the same lines as previously starting with a similar estimate as (25) for $1<s \leq 2$.

The next lemma is needed in the two dimensional case when $\alpha>2$.

Proposition 5.2. Let $\Omega \subset \mathbb{R}^{2}$ and $\Gamma_{C} \subset \partial \Omega$. Consider $v \in V \cap H^{2}(\Omega)$ and $v_{h} \in V_{h}$. Assume that $h$ is small enough. Then

$$
\left\|v-v_{h}\right\|_{L^{\infty}\left(\Gamma_{C}\right)} \lesssim h \sqrt{-\ln (h)}|v|_{H^{2}(\Omega)}+\sqrt{-\ln (h)}\left\|v-v_{h}\right\|_{H^{1}(\Omega)} .
$$

Proof. We start with the triangular inequality $\left\|v-v_{h}\right\|_{L^{\infty}\left(\Gamma_{C}\right)} \leq\left\|v-\pi_{h} v\right\|_{L^{\infty}\left(\Gamma_{C}\right)}+\| \pi_{h} v-$ $v_{h} \|_{L^{\infty}\left(\Gamma_{C}\right)}$ where $\pi_{h}$ is the Lagrange interpolator mapping into $V_{h}$ and we consider separately $\left\|v-\pi_{h} v\right\|_{L^{\infty}\left(\Gamma_{C}\right)}$ and $\left\|\pi_{h} v-v_{h}\right\|_{L^{\infty}\left(\Gamma_{C}\right)}$.

From the Gagliardo-Nirenberg interpolation inequality in one dimension (see [3]), standard error estimates (e.g., issued from Proposition 5.1) and the trace theorem, we get

$$
\begin{aligned}
\left\|v-\pi_{h} v\right\|_{L^{\infty}\left(\Gamma_{C}\right)} & \lesssim\left\|v-\pi_{h} v\right\|_{L^{2}\left(\Gamma_{C}\right)}^{1 / 2}\left\|v-\pi_{h} v\right\|_{H^{1}\left(\Gamma_{C}\right)}^{1 / 2} \\
& \lesssim\left(h^{3 / 2}|v|_{H^{3 / 2}\left(\Gamma_{C}\right)} h^{1 / 2}|v|_{H^{3 / 2}\left(\Gamma_{C}\right)}\right)^{1 / 2} \\
& \lesssim h|v|_{H^{2}(\Omega)} .
\end{aligned}
$$

Consider now the discrete term $\left\|\pi_{h} v-v_{h}\right\|_{L^{\infty}\left(\Gamma_{C}\right)}$. Apply the one dimensional global inverse inequality on $\Gamma_{C}$ with $p \in[1, \infty]([15])$, and obtain

$$
\left\|\pi_{h} v-v_{h}\right\|_{L^{\infty}\left(\Gamma_{C}\right)} \lesssim h^{-1 / p}\left\|\pi_{h} v-v_{h}\right\|_{L^{p}\left(\Gamma_{C}\right)} .
$$

Then, using the embedding inequality $\|v\|_{L^{p}\left(\Gamma_{C}\right)} \lesssim \sqrt{p}\|v\|_{H^{\frac{1}{2}\left(\Gamma_{C}\right)}}$ for all $v \in H^{1 / 2}\left(\Gamma_{C}\right)$ and $p \in[1, \infty)$ (see $[2])$, it follows

$$
\left\|\pi_{h} v-v_{h}\right\|_{L^{\infty}\left(\Gamma_{C}\right)} \lesssim \sqrt{p} h^{-1 / p}\left\|\pi_{h} v-v_{h}\right\|_{H^{1 / 2}\left(\Gamma_{C}\right)} .
$$

Choosing $p=-\ln (h)$ yields

$$
\left\|\pi_{h} v-v_{h}\right\|_{L^{\infty}\left(\Gamma_{C}\right)} \lesssim \sqrt{-\ln (h)}\left\|\pi_{h} v-v_{h}\right\|_{H^{1}(\Omega)} .
$$

Finally, using a triangular inequality and Proposition 5.1, we obtain

$$
\left\|\pi_{h} v-v_{h}\right\|_{L^{\infty}\left(\Gamma_{C}\right)} \lesssim h \sqrt{-\ln (h)}|v|_{H^{2}(\Omega)}+\sqrt{-\ln (h)}\left\|v-v_{h}\right\|_{H^{1}(\Omega)} .
$$

The result then follows by collecting these estimates and observing that $h \lesssim h \sqrt{-\ln (h)}$ for $h$ small enough. 


\section{References}

[1] R.A. Adams. Sobolev spaces. Academic Press, New York-London, 1975. Pure and Applied Mathematics, Vol. 65.

[2] F. Ben Belgacem. Numerical simulation of some variational inequalities arisen from unilateral contact problems by the finite element methods. SIAM J. Numer. Anal., 37(4):1198-1216, 2000.

[3] H. Brezis. Functional analysis, Sobolev spaces and partial differential equations. Springer, 2010.

[4] E. Burman, P. Hansbo, and M.G. Larson. The penalty-free Nitsche method and nonconforming finite elements for the Signorini problem. SIAM J. Numer. Anal., 55(6):173-195, 2017.

[5] E. Burman, P. Hansbo, and M.G. Larson. Augmented lagrangian finite element methods for contact problems. ESAIM-Mathematical Modelling and Numerical Analysis, 53(1):2523-2539, 2019.

[6] F. Chouly and P. Hild. A Nitsche-based method for unilateral contact problems: numerical analysis. SIAM J. Numer. Anal., 51(2):1295-1307, 2013.

[7] F. Chouly and P. Hild. On convergence of the penalty method for unilateral contact problems. Applied Numerical Mathematics, 65:27-40, 2013.

[8] F. Chouly, P. Hild, and Y. Renard. Symmetric and non-symmetric variants of Nitsche's method for contact problems in elasticity: theory and numerical experiments. Math. Comp., 84(293):1089-1112, 2015.

[9] C. Christof and C. Haubner. Finite element error estimates in non-energy norms for the two-dimensional scalar Signorini problem. Preprint No. IGDK-2018-14; August 2018, 2018.

[10] P. Coorevits, P. Hild, K. Lhalouani, and T. Sassi. Mixed finite element methods for unilateral problems: convergence analysis and numerical studies. Math. Comp., 71(237):1$25,2002$.

[11] I. Dione. Optimal convergence analysis of the unilateral contact problem with and without Tresca friction conditions by the penalty method. J. Math. Anal. Appl., 472(1):266$284,2019$.

[12] I. Dione. Optimal error estimates of the unilateral contact problem in a curved and smooth boundary domain by the penalty method. IMA Journal of Numerical Analysis, 40(1):729-763, 2020.

[13] G. Drouet and P. Hild. Optimal convergence for discrete variational inequalities modelling Signorini contact in $2 \mathrm{~d}$ and $3 \mathrm{~d}$ without additional assumptions on the unknown contact set. SIAM J. Numer. Anal., 53(3):1488-1507, 2015. 
[14] G. Drouet and P. Hild. An accurate local average contact method for nonmatching meshes. Numer. Math., 136(2):467-502, 2016.

[15] A. Ern and J.-L. Guermond. Theory and practice of finite elements, volume 159 of Applied Mathematical Sciences. Springer-Verlag, New York, 2004.

[16] R. Glowinski, J.-L. Lions, and R. Trémolières. Numerical analysis of variational inequalities, volume 8 of Studies in Mathematics and its Applications. North-Holland Publishing Co., Amsterdam-New York, 1981. Translated from the French.

[17] R. Glowinski and A. Marroco. Sur l'approximation, par éléments finis d'ordre un, et la résolution, par pénalisation-dualité d'une classe de problèmes de Dirichlet non linéaires. ESAIM: Mathematical Modelling and Numerical Analysis - Modélisation Mathématique et Analyse Numérique, 9(R2):41-76, 1975.

[18] P. Grisvard. Elliptic problems in nonsmooth domains, volume 69 of Classics in Applied Mathematics. Society for Industrial and Applied Mathematics (SIAM), Philadelphia, PA, 2011.

[19] H. Hardering. The Aubin-Nitsche trick for semilinear problems. arXiv:1707.00963, 2017.

[20] P. Hild and Y. Renard. A stabilized lagrange multiplier method for the finite element approximation of contact problems in elastostatics. Numer. Math., 115(1):101-129, 2010.

[21] S. Hueber and B. Wohlmuth. An optimal a priori error estimate for nonlinear multibody contact problems. SIAM J. Numer. Anal., 43:156-173, 2005.

[22] N. Kikuchi and J. T. Oden. Contact problems in elasticity: a study of variational inequalities and finite element methods, volume 8 of SIAM Studies in Applied Mathematics. Society for Industrial and Applied Mathematics (SIAM), Philadelphia, PA, 1988.

[23] A. Klarbring, A. Mikelic, and M. Shillor. Frictional contact problems with normal compliance. Internat. J. Engrg. Sci., 26:811-832, 1988.

[24] A. Klarbring, A. Mikelic, and M. Shillor. On friction problems with normal compliance. Nonlinear Anal., 13:935-955, 1989.

[25] J.A.C. Martins and J.T. Oden. Existence and uniqueness results for dynamic contact problems with nonlinear normal and friction interface laws. Nonlinear Anal., 11:407428, 1987.

[26] Z. Mghazli. Regularity of an elliptic problem with mixed Dirichlet-Robin boundary conditions in a polygonal domain. Calcolo, 29(3-4):241-267 (1993), 1992.

[27] F. Natterer. Optimale L2-konvergenz finiter elemente bei variationsungleichungen. Bonner Math. Schriften, 89:1-12, 1976. 
[28] J.T. Oden and J.A.C. Martins. Models and computational methods for dynamic friction phenomena. Comput. Methods. Appl. Mech. Engrg., 52:527-634, 1987.

[29] G. Savaré. On the regularity of the positive part of functions. Nonlinear Anal., 27(9):1055-1074, 1996.

[30] O. Steinbach, B. Wohlmuth, and L. Wunderlich. Trace and flux a priori error estimates in finite element approximations of Signorini-type problems. IMA J. Numer. Anal., 36(3):1072-1095, 2016. 